\title{
Transformative Lehre für eine kritisch-geographische (Berufs-)Praxis
}

\author{
Katja Thiele ${ }^{1}$ Sören Becker ${ }^{1}$
}

Eingegangen: 30. März 2020 / Überarbeitet: 9. September 2020 / Angenommen: 10. September 2020 / Online publiziert: 7. Oktober 2020

(c) Der/die Autor(en) 2020

\section{Zusammenfassung}

Der Beitrag entwickelt die Perspektive einer kritischen Lehre zum Thema Nachhaltigkeit und zeigt deren Relevanz für die spätere Berufspraxis von Geograph*innen auf. Es wird diskutiert, inwiefern ein komplexes und kritisches Verständnis von Nachhaltigkeit zielführend zu einer transformativen Lehrpraxis weiterentwickelt werden kann. Kritische Lehre entsteht aus unserer Sicht aus der Verbindung von drei zentralen Merkmalen: Seminarinhalte, die gesellschaftliche Machtverhältnisse hinterfragen; Lernformen, die eine Selbstreflexion der Studierenden ermöglichen; und die Zusammenarbeit mit transformativen Initiativen aus der Praxis. Der Beitrag beleuchtet auf der Grundlage der Erfahrungen eines Projektseminars sowohl die Potenziale als auch die Spannungsfelder und Widersprüche dieses Ansatzes.

Schlüsselwörter Transformation $\cdot$ Kritische Lehre $\cdot$ Berufspraxis $\cdot$ Nachhaltigkeit $\cdot$ Bildung für nachhaltige Entwicklung

\section{Transformative teaching for critical geographical (professional) practice}

\begin{abstract}
This article develops a perspective on critical teaching in the field of sustainability, and shows its relevance to the professional practice of geographers. We discuss how a complex and critical understanding of sustainability may be usefully developed for a transformative teaching practice. From our perspective, critical teaching consists of three core elements: seminar content that questions social power relations, didactical methods that enable the students to reflect, and collaboration with transformative initiatives from outside the University. Based on experience from a project seminar, the article sheds light on the potential as well as the tensions and contradictions of this approach.
\end{abstract}

Keywords Transformation - Critical teaching $\cdot$ Professional practice $\cdot$ Sustainability $\cdot$ Education for sustainable development

\section{Einleitung}

Das Feld der Nachhaltigkeit ist nicht nur ein wichtiges Forschungsthema für die Geographie, sondern auch seit 30 Jahren ein stetig wachsender Arbeitsmarkt für GeographieStudierende. Als Nachhaltigkeitsbeauftragte oder Bildungsreferenten sind Absolvent*innen in Unternehmen, Regie-

Katja Thiele

kthiele@uni-bonn.de

Sören Becker

soeren.becker@uni-bonn.de

1 Geographisches Institut, Universität Bonn, Meckenheimer Allee 166, 53115 Bonn, Deutschland rungs- oder Nichtregierungsorganisationen beschäftigt (Daten zur geographischen Berufspraxis Hünnemeyer 2019). Sie entwickeln Nachhaltigkeitskonzepte, setzen Planungsstrategien um oder beraten Unternehmen, Bildungseinrichtungen und Organisationen bei der Erstellung von Nachhaltigkeitsstrategien. Nachhaltige Entwicklung ist dabei nicht nur auf den Globalen Süden, sondern auch das geographische „Nahfeld“ bezogen.

Den Hochschulen kommt als Bildungsinstitutionen eine besondere Verantwortung zu, aktuelle Herausforderungen zu thematisieren. Die herausragende Rolle der Geographie im Nachhaltigkeitsfeld wird neben vielfältigen Forschungsaktivitäten (u. a. Schulz 2012, Schwanen 2018) und der Akzentuierung von Nachhaltigkeitsthemen in der Lehre (u.a. Leal Filho 2018, S. 2) auch institutionell in der Koordi- 
nation eines internationalen Wissenschaftsjahres zum Thema durch das Geographische Institut der Friedrich-SchillerUniversität Jena sichtbar. Eine intensive fachwissenschaftliche Debatte über die Anforderungen und Merkmale einer Bildung für nachhaltige Entwicklung findet jedoch erst in den letzten 10 Jahren statt und ist in der Lehrpraxis bisher noch zu wenig verankert (Coy 2007; Zimmermann und Zimmermann-Janschitz 2016).

Dieser Beitrag diskutiert die Möglichkeiten einer kritischen Hochschullehre im Feld Nachhaltigkeit als berufliche Vorbereitung zukünftiger Nachhaltigkeitspraktiker*innen. Mit Rückgriff auf Debatten in der Fachdidaktik und der Kritischen Geographie wird der Unterschied zwischen einem instrumentellen und kritisch-emanzipatorischen Verständnis von Bildung herausgearbeitet. Daraus werden Merkmale einer transformativen Lehrpraxis abgeleitet und anhand eines Projektseminars zum Thema „Alternatives Wirtschaften in der Region“ diskutiert. Der Artikel schließt mit einem Blick auf die sich daraus ergebenden Potenziale, Spannungsfelder und Widersprüche.

\section{Nachhaltigkeit in der geographischen Hochschullehre}

Es gilt heute beinahe als Selbstverständlichkeit, dass Absolvent*innen der Geographie über konzeptionelle und praktische Kompetenzen im Bereich Nachhaltigkeit verfügen. Eine Schwierigkeit besteht jedoch darin, dass mit dem Nachhaltigkeitsbegriff eine Vielzahl von Konzepten verbunden ist. Sinnbildlich dafür steht die populäre Darstellung des „Nachhaltigkeitsdreiecks“, das ökologische, soziale und ökonomische Dimensionen von Nachhaltigkeit zu verbinden sucht (Pufé 2018, S. 112f.). Nachhaltigkeit impliziert ein komplexes, ganzheitliches Verständnis sozialer Prozesse. Wie sich diese Orientierung auf Komplexität und Widerstände in der geographischen Lehre vermitteln lässt, wird seit mehr als zwei Jahrzehnten in der Fachdidaktik diskutiert. Vor allem vier Kompetenzen werden hervorgehoben: das Verstehen von Systemen und deren komplexe Interaktion (Vester 2002), die aktive Integration verschiedener disziplinärer Zugänge (Hauenschild und Bolscho 2006) sowie die Förderung der Fähigkeiten, einerseits Divergenzen bzw. Kontroversen aufzuspüren und andererseits, ,transdisziplinär“ und ,synergieorientiert“ im sozialen Austausch Lösungen zu entwickeln (Krol 2006). Die Rolle und Form von Lehre und die Kombination von Theorie und Praxis scheinen in den fachwissenschaftlichen Diskussionen um sozialökologische Transformationen und transformative Wissenschaft jedoch immer noch ein eher randständiges Thema zu sein (Schneidewind 2015) - und dass, obwohl es notwendig ist, intensiver darüber nachzudenken, wie gerade komplexe Themen wie Nachhaltigkeit,
Klimawandel oder die Corona-Krise vermittelt werden können. Schneider et al. (2018) sprechen von einem ,TeachingResearch-Practice-Nexus“, d.h. die drei Bereiche Lehre, Forschung und Praxis sind eng miteinander verbunden, lassen sich aber nicht reibungslos ineinander übersetzen.

Andere Autor*innen sehen in der erhöhten Aufmerksamkeit für Nachhaltigkeitsthemen jedoch ein Problem. Durch den oft unscharfen Gebrauch wird in der Didaktik ein „Aushöhlen“ (hollowing out; Grober 2015, S. 6f.) des Begriffs der Nachhaltigkeit befürchtet. Diese Tendenz werde in der Lehre verstärkt, da der abstrakte Begriff der Nachhaltigkeit eine Bezugnahme auf verschiedene Themenbereiche (z. B. politische Rahmenbedingungen, ökonomische Globalisierung) erfordert und den Studierenden gleichzeitig die praktische Anwendbarkeit sowie der persönliche Alltagsbezug vermittelt werden müssen (Leal Filho 2018, S. 7; Zimmermann und Zimmermann-Janschitz 2016, S. 140). Das hat zur Folge, dass Studierende aus diversen theoretischen Perspektiven und vor dem Hintergrund verschiedener Dimensionen von Nachhaltigkeit zu völlig konträren Schlüssen kommen können (Coy 2007, S. 9; Pufé 2018, S. 112f.).

Für Getzin und Singer-Brodowski (2016) birgt die verstärkte Diskussion um Bildung für Nachhaltige Entwicklung zudem ,die Gefahr, dass kritischere Perspektiven ... marginalisiert [werden] und [dies] zu einer De-Politisierung ökologischer und sozialer Debatten beiträgt" (Getzin und Singer-Brodowski 2016, S. 37). Sie unterscheiden daher zwischen einer instrumentellen Bildung für nachhaltige Entwicklung und einer kritisch-emanzipatorischen Bildung als nachhaltige Entwicklung. Während der instrumentelle Ansatz die Vermittlung von „ExpertInnenwissen zur sozial-ökologischen Transformation“ (ebd.) in den Mittelpunkt rücke und die Grundlage für das Verständnis für Handlungsoptionen und Lösungsansätze lege, stelle der kritisch-emanzipatorische Ansatz die Diskussion über Werte, Leitbilder und Normen dieser Transformation(en) ins Zentrum. Kritisch-emanzipatorische Bildung als nachhaltige Entwicklung kann verstanden werden als aktivierende, hinterfragende Lehrpraxis, die auf die Persönlichkeitsentwicklung der Studierenden fokussiert. Das „Ziel eines nachhaltigen Verhaltens ... wird ... dem Ziel der Entwicklung einer kritischen Reflexionsfähigkeit und autonomen Entscheidungs- und Handlungsfähigkeit der Lernenden untergeordnet“" (ebd.). Diese Lehrpraxis setzt voraus, Seminarinhalte, Lernformen und praktische Perspektiven für eine gesellschaftliche Transformation zu kombinieren und erfordert von Dozierenden die Kompetenz, mit den Studierenden im offenen Austausch Lösungen zu diskutieren und gemeinsam (weiter-)zuentwickeln (Krol 2006). 


\section{Konzeptionen transformativer Lehre}

In diesem Abschnitt entwickeln wir mit Rückgriff auf Diskussionen in der Kritischen Geographie (u. a. Belina 2008) Merkmale einer kritischen Nachhaltigkeitsdidaktik (Inhalte, Form und transformative Perspektive).

Die Seminarinhalte sollten eine kritische Auseinandersetzung mit der Komplexität und Vielfalt des Nachhaltigkeitsbegriffs beinhalten. Existierende geographische Konzepte zur Erklärung gesellschaftlicher Machtverhältnisse und ungleicher Entwicklung können diskutiert und, auf Nachhaltigkeitsfragen bezogen, weiterentwickelt werden. Ein Hemmnis für die Lehre radikal-kritischer Konzepte (u. a. Postwachstumsgeographien, Lange et al. 2020; Imperiale Lebensweise, Brand und Wissen 2017) besteht für Leal Filho (2018, S. 6) darin, dass die Thematisierung von Widersprüchen in der Lehre Spannungen zwischen Dozierenden und Studierenden, unter den Studierenden und im Austausch mit externen Akteuren hervorbringe. Um Reflexionen über die Rolle der Studierenden als spätere Praktiker*innen anzuregen, sind aber gerade die Vermittlung der Fähigkeiten des kritischen Denkens und (Selbst-)Reflexion wichtig (Van der Schee 2003).
Dafür bedarf es neben den Seminarinhalten einer didaktischen Lehrform, die Widersprüchen einen Raum gibt und „durch Reflexion ..., echten Dialog ... und flexible Strukturen“ gekennzeichnet ist (Leal Filho 2018, S. 6). Eine Lehre, die über die reine Wissensvermittlung hinausgeht (Bürk 2016), kann erreicht werden, wenn genügend Zeit für ergebnisoffene Diskussionen und die Aushandlung von Widersprüchen zwischen Nachhaltigkeitsperspektiven und den dadurch möglicherweise auftretenden Konflikten eingeräumt wird. Da sich Zielkonflikte nicht vermeiden lassen, ist es wichtig, sie selbst zum Gegenstand des Lernens zu machen: durch flexible und kleingruppenbezogene Formate sowie strukturierte Diskussionen und das Zulassen von Konflikten. Hier kann zurückgegriffen werden auf offene und aktivierende Diskussionsformate (beispielsweise Fishbowl-Diskussionen, Lamker et al. 2018).

Eine transformative Perspektive in der Lehre entsteht erst durch die Verknüpfung von Hochschullehre mit aktuellen gesellschaftlichen Konflikten und den potenziellen Transformationen. Heilgemeir et al. (2016) schlagen dafür eine Lehre im Dialog mit Akteuren aus der ,alternativen“ Praxis vor. Das heißt, es werden aktiv Initiativen und außeruniversitäre Akteure in die Lehre eingebunden (durch Vorträge, Feldbesuche, Projektarbeiten) und gemeinsam adäqua-
Abb. 1 Transformative Lehre. (Quelle: eigene Darstellung in Anlehnung an Maschkowsky und Wanner 2014, S. 3)

\section{TRANSFORMATIVE LEHRE \\ (am Beispiel eines Projektseminares zu alternativem $W_{\text {irtschaften) }}$}

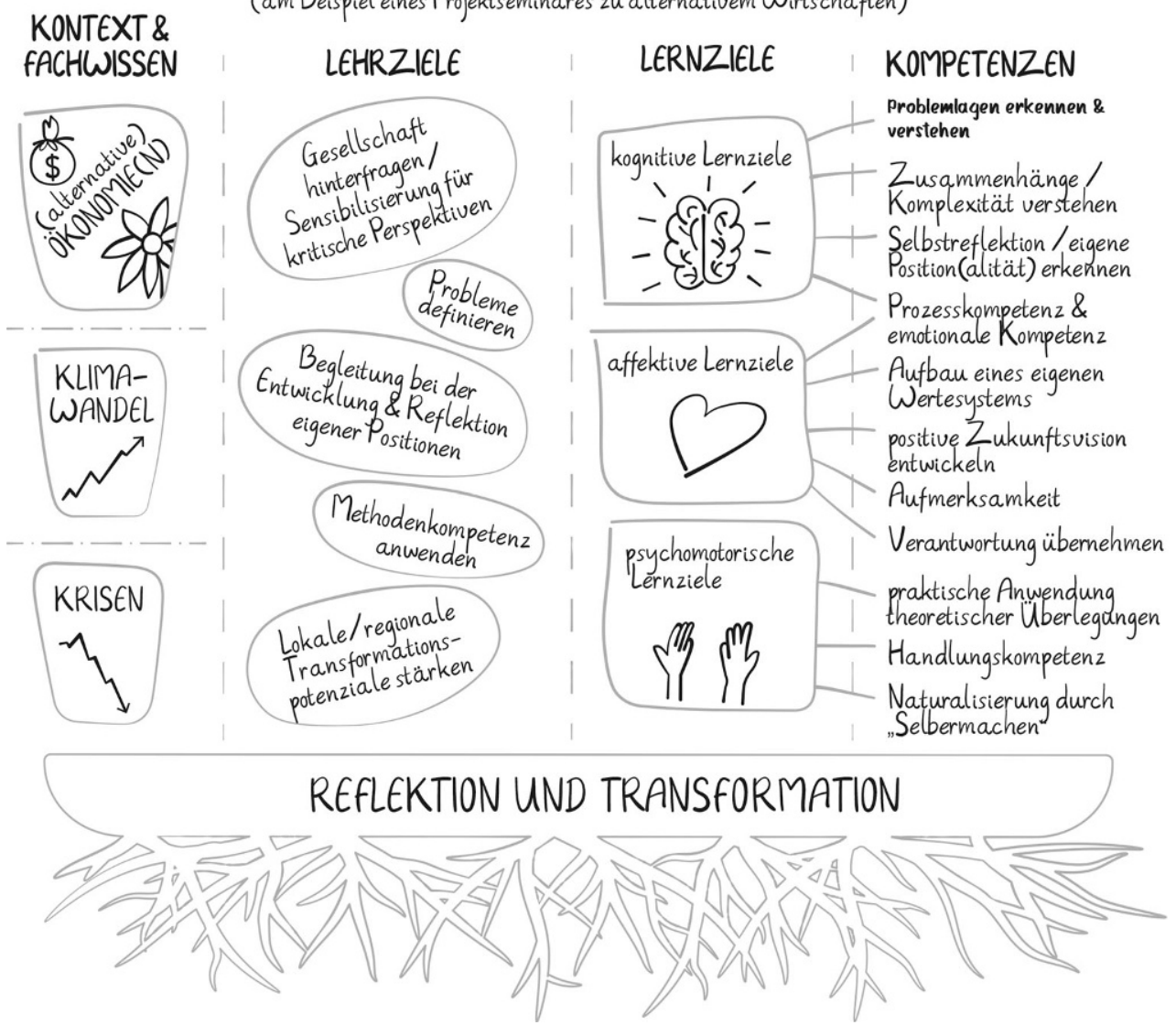




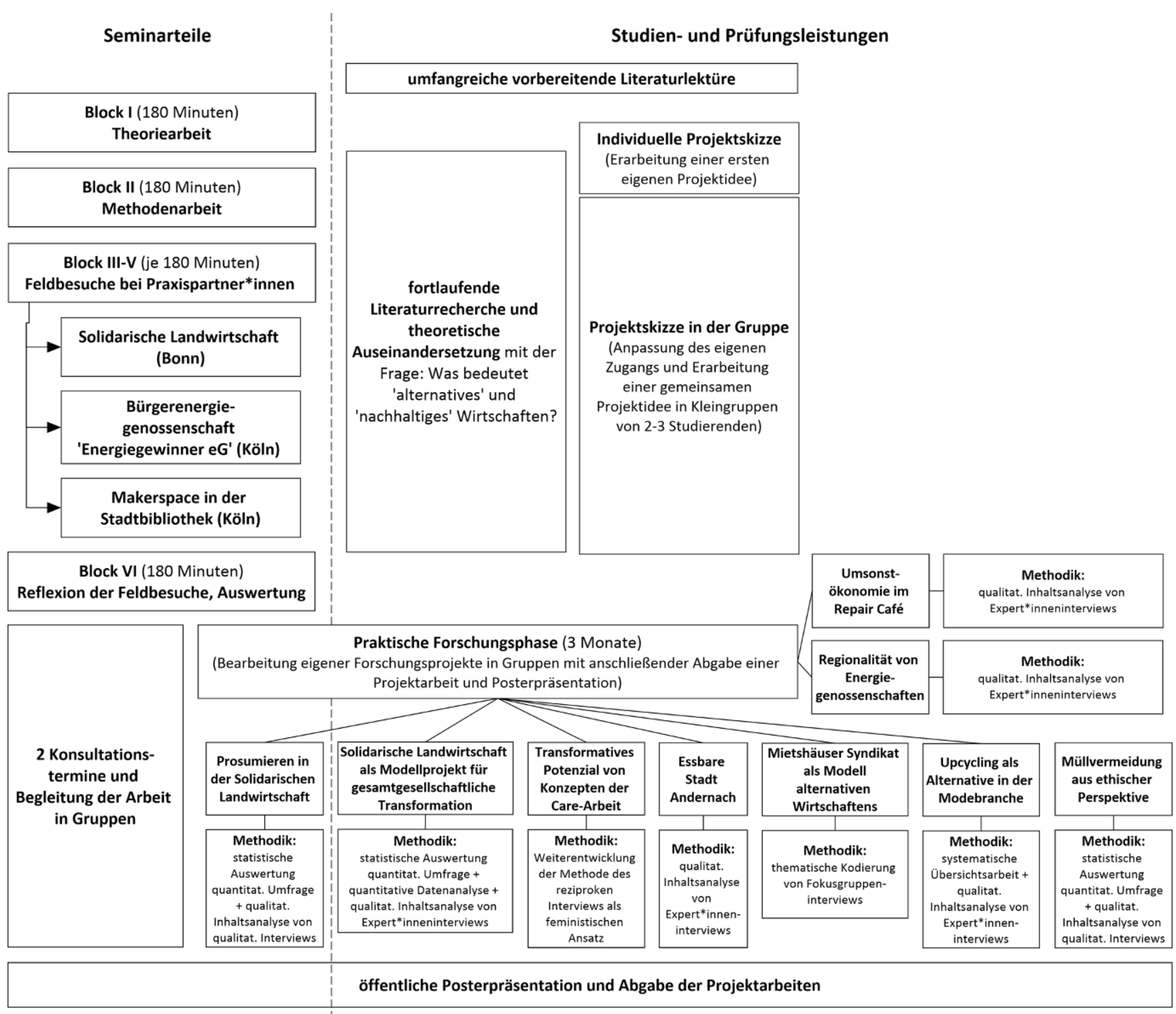

Abb. 2 Umsetzung des Lehrkonzeptes im Projektseminar. (Quelle: eigene Darstellung)

te Lehrformate entwickelt. Empfohlen wird die Diskussion verschiedener Nachhaltigkeitsansätze, mit Fokus auf noch junge, im öffentlichen und wissenschaftlichen Diskurs bisher marginalisierte Positionen (beispielsweise von selbstorganisierten Initiativen, kleinen gemeinnützig agierenden Unternehmen). Neben dem Erwerb praxisrelevanten Wissens trägt die Diskussion der Widersprüche innerhalb und gegenüber solchen Projekten dazu bei, die Reflexions- und Kritikkompetenzen der Studierenden zu schärfen.

\section{Projektseminar „Alternatives Wirtschaften“}

Als Beispiel für die Umsetzung dieser Prinzipien wird nun die Konzeption des Projektseminars „Alternatives Wirtschaften in der Region“ vorgestellt, das wir im Win- tersemester 2018/19 an der Universität Bonn durchgeführt haben (Abb. 1 und 2). Die Leistungsanforderungen umfassen die Durchführung eines wissenschaftlichen Forschungsprojektes in kleinen Gruppen, die Vorstellung des Zwischenstandes (Konsultationstermin), eine öffentliche Poster-Präsentation der ersten Ergebnisse im Geographischen Institut und eine anschließend einzureichende Projektarbeit, die das Forschungsprojekt und die Ergebnisse darstellt (Abb. 2). Das Seminar wurde durch eine Evaluation mithilfe teilstandardisierter Fragebögen begleitet, in dem die Studierenden 3-mal während des Seminarverlaufs ihre Erfahrungen und Kompetenzgewinne selbst einschätzen sollten. In diesem Beitrag geht es weniger um die Darstellung einer Best Practice, sondern um eine Verdeutlichung der Möglichkeiten und der praktischen Widersprüche, die bei einer kritischen Nachhaltigkeitslehre entstehen können. 
Die transformative Lehre, wie sie vorhergehend eingeführt wurde, benötigt eine starke Verknüpfung von Theorie, Methodik und Praxis. Das Seminar wurde dafür in einen Theorie-, einen Methodenblock, drei Praxisblöcke sowie einen Reflexionsblock unterteilt, die jeweils zeitlich geblockt wurden. Den Lerntaxonomien von Bloom (1972) und Kratwohl et al. (1975) folgend, wurde zudem darauf geachtet, sowohl kognitive als auch affektive und psychomotorische Lernziele anzusprechen (Abb. 1).

Zentrale Inhalte des Seminars betrafen die Analyse der Wachstumsorientierung von Wirtschaft und Gesellschaft sowie alternativer Unternehmensformen und Wege der Bereitstellung von Dienstleistungen (Schmid und Smith 2020). Neben der Vermittlung von Kontext- und Fachwissen zu wesentlichen inhaltlichen Aspekten (u.a. Widersprüche zwischen Wachstumsorientierung und wiederkehrenden Krisen, Externalisierung ökologischer Kosten durch Ressourcenabbau, Emissionen, Klimawandel; Abb. 1) stand die gemeinsame Entwicklung einer Systematik zur Analyse von „Praktiken alternativen Wirtschaftens“ im Mittelpunkt des Seminars. Zur Vorbereitung auf den Theorieblock erhielten die Studierenden im Vorfeld umfangreiche Lektüre sowie Fragen. Anhand der Texte wurden vier Untersuchungsdimensionen (Entstehung \& Akteure, Geschäftsmodell \& Profit, Organisationsformen \& Entscheidungen, Lieferketten \& räumliche Beziehungen) erarbeitet. Mit deren Hilfe konnten in der Folge die Praxispartner auf ihre „Alternativität" hin beurteilt werden.

Die Seminarform war durch offene Diskussionen sowie die selbstständige Reflexion der Studierenden charakterisiert. Die Einschätzung von Alternativität diente nicht dazu, die jeweiligen Perspektiven als ,richtig“ oder ,falsch“ zu bewerten, sondern mit den Studierenden zu diskutieren, nach welchen Wertmaßstäben und innerhalb welcher Grenzen Alternativität und Nachhaltigkeit bewertet werden können. Es wurde außerdem viel Zeit darauf verwendet, im Seminar Gruppen zu bilden, Fragestellungen zu entwickeln und die Studierenden dazu zu befähigen, geeignete Methoden für ihre Forschungsfragen zu wählen. Als hilfreich erwies sich, dass das Seminar mit zwei Dozierenden stattfand.

Der Bezug zur transformativen Praxis wurde auf zwei Ebenen hergestellt: erstens, durch drei Feldbesuche bei Praxispartner*innen aus der Region (solidarische Landwirtschaft in Bonn, Energiegenossenschaft „Energiegewinner“ in Köln, Makerspace der Stadtbibliothek in Köln; Abb. 2). Die ausgewählten Projekte sollten sich hinsichtlich ihrer Organisationsformen (selbstorganisiert, genossenschaftlich, staatlich) unterscheiden und alle drei Dimensionen von Nachhaltigkeit (auf je andere Weise) ansprechen. Vor Ort wurde mit den Praxispartner*innen über die Fragen diskutiert, die sich für die Studierenden aus den Untersuchungsdimensionen ergaben.

Zweitens folgte auf den Präsenzteil des Seminars eine praktische Forschungsphase, in der die Gruppen selbstständig ihre Forschungsprojekte durchführten. Im Sinne einer transformativen Wirkung der Lehre in die Praxis wurde die gemeinsame Entwicklung von Fragestellungen zwischen Studierenden und (weiteren) Praxispartner*innen angestrebt. Die Frage, ob und wie sich wissenschaftliche Erkenntnisse zielführend in die Praxis übersetzen lassen, hat die Studierenden besonders begleitet. Während dieser Phase wurden zwei Konsultationstermine angeboten, die als große Hilfe empfunden wurden und zur Qualität der Ergebnisse beigetragen haben.

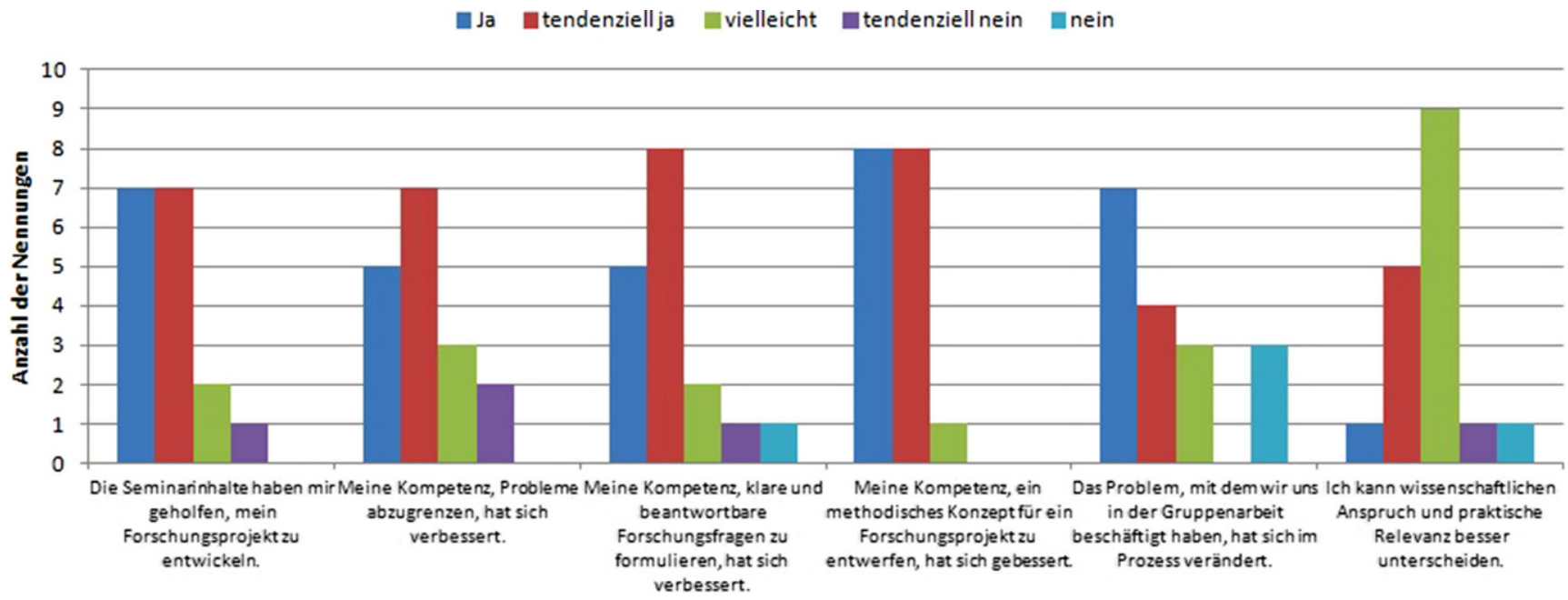

Abb. 3 Ergebnisse der 3. Selbsteinschätzung zum Ende des Seminars. (Quelle: eigene Darstellung) 


\section{Spannungsfelder transformativer Lehre}

Das Seminar ist von den Studierenden sehr gut aufgenommen worden. Sowohl in der regelmäßigen Selbsteinschätzung des Lernfortschritts als auch im direkten Feedback hat sich gezeigt, dass große Lernfortschritte in der Anwendung methodischer Kenntnisse sowie bei der Verknüpfung von Theorie und Methodik gemacht wurden (Abb. 3). In der abschließenden Veranstaltungsevaluation haben zudem 88,9\% der Studierenden angegeben, dass die Lehrform da$\mathrm{zu}$ beigetragen hat, auch schwierige Inhalte verständlich zu vermitteln und eine selbstständige, aktive Auseinandersetzung mit den Lerninhalten zu fördern.

Während die Studierenden im gemeinsamen Seminarteil sehr offen für kritische Themen waren und bereits über ein solides methodisches Grundwissen verfügten, ergaben sich Spannungen vor allem in der Interaktion mit den Praxispartner*innen, d.h. der Verbindung von Inhalt, Form und transformativer Perspektive. Vor allem die Unterscheidung und Verknüpfung von wissenschaftlichem Anspruch und praktischer Relevanz ihrer Arbeit fiel den Studierenden bis zuletzt nicht leicht (Abb. 3).

Erstens besteht ein Spannungsfeld im ambivalenten Verhältnis von wissenschaftlicher Methodik und den Bedürfnissen und Interessen der Initiativen in der Praxis. Der Dialog mit den lokalen Initiativen war Inspiration und Herausforderung zugleich. Obwohl er den meisten Teilnehmenden leicht fiel und als Bereicherung empfunden wurde, kam es bei der Definition von Problemen und der Entwicklung von Projekten und Zielen zu Konflikten und Abstimmungsproblemen mit den Initiativen. Eine Studierende beschreibt, wie im Abstimmungsprozess mit der Initiative klar wurde, „dass die eigenen Forschungsziele und -fragen nicht unbedingt zu den Zielen der Initiativen passen" (Zitat Evaluation).

Zweitens hat sich der transparente Umgang mit den Möglichkeiten und Grenzen, im Seminar die Komplexität von Nachhaltigkeitsproblemen zu erfassen, als wichtig erwiesen. Dadurch konnten bei den Studierenden entscheidende Reflexionsprozesse ausgelöst werden: „Es wurde viel über Möglichkeiten und Grenzen gesprochen, was half, dies auf die eigene Forschung zu übertragen" (Zitat Evaluation). Durch die immanente Verunsicherung konnten die Studierenden einerseits ein Verständnis für das Verhältnis von wissenschaftlicher Theorie und Empirie entwickeln und andererseits wurden sie sensibilisiert für die Logiken und Widersprüche von Theorie, Methodik und Praxis.

Die Schwierigkeiten der Studierenden, mit diesen Widersprüchen im Projekt umzugehen, sind Teil des transformativen Lernprozesses, müssen aber intensiv begleitet werden, um den Lernerfolg sicherzustellen. Das Einfangen individueller Schwierigkeiten stellt Lehrende vor Herausforderungen (u. a. Widersprüche zwischen Forschung und Praxis an- tizipieren und darstellen, Komplexität in Diskussionen vermitteln, hoher Beratungsaufwand bei Problemen). Dennoch ist gerade das Aushalten dieser Spannungen wichtig. Es hängt erheblich vom Projektverlauf und von der Feedbackkultur ab, inwieweit Studierende ihre Position in der Welt, innerhalb und außerhalb der Universität reflektieren und eine positive Zukunftsvision entwickeln (können), an der sie partizipieren möchten (Abb. 1). So hat beispielsweise ein Student erlebt, ,dass tiefgehende Kapitalismuskritik außerhalb der Uni stattfindet!“ (Zitat Evaluation). Erst die Auseinandersetzung mit der für ihn kapitalismuskritischen Praxis und die Weiterentwicklung seiner (Selbst-)Reflexionskompetenz ermöglichte es ihm, Verknüpfungen zwischen verschiedenen Initiativen zu erkennen, sich selbst ins Verhältnis dazu zu setzen und Rückschlüsse für die eigene $\mathrm{Zu}$ kunft zu ziehen.

\section{Schlussfolgerungen}

Dieser Beitrag hat den Stellenwert geographischer Lehre für die spätere Berufspraxis von Geographie-Studierenden diskutiert. Damit kann sie einen wichtigen Beitrag für eine allgemeine gesellschaftliche Nachhaltigkeitstransformation leisten. Die Umsetzung einer transformativen Lehrpraxis im Sinne einer Bildung als nachhaltige Entwicklung hilft bei der Vermittlung komplexer Problemlagen und fördert zahlreiche Kompetenzen bei den Studierenden, die in der späteren Berufspraxis hochrelevant sind. Sie ist jedoch auch ressourcen- und zeitintensiv und findet im universitären Alltag ihre Grenzen.

Am Beispiel einer eigenen Lehrveranstaltung haben wir Merkmale und Herausforderungen einer kritischen Lehre im Nachhaltigkeitsfeld diskutiert. Eine transformative Lehrpraxis erfordert aus unserer Sicht die zusammenhängende Entwicklung von Seminarinhalten, Lehr- und Lernformen und einer praktischen Perspektive auf die Nachhaltigkeitstransformation. Das setzt voraus, einen Umgang mit der Mehrdeutigkeit des Nachhaltigkeitsbegriffs zu finden und die dahinterliegenden gesellschaftlichen Machtverhältnisse zu diskutieren. Die didaktische Methodik sollte sich nicht auf die reine Vermittlung von Fachwissen beschränken, sondern Studierenden ermöglichen, ihre eigene Position zu finden. Um die hierfür notwendigen kognitiven und affektiven Kompetenzen zu stärken, braucht es Zeit und Raum für ergebnisoffene Diskussionen sowie das Zulassen von Spannungen und echtem Dialog. Letztlich hat sich gezeigt, dass ein intensiver Dialog mit der ,alternativen“ Praxis zwar herausfordernd ist, aber sehr viel Potenzial bietet, um den Studierenden ein Verständnis für das komplexe Verhältnis von Theorie, Methodik und Praxis zu ermöglichen.

Studierende können auf diesem Weg auch für die Widersprüche und Konflikte einer Berufspraxis im Feld der 
Nachhaltigkeit sensibilisiert werden. Sie lernen Konzepte und Theorien kennen und erwerben Wissen über alternative Handlungsmöglichkeiten und deren Grenzen. Die geschulte Urteilsfähigkeit hilft nicht nur in der Entwicklung von Nachhaltigkeitsthemen im Berufsalltag, sondern auch bei der Auseinandersetzung mit hochaktuellen Tendenzen der Wissenschaftsfeindlichkeit.

Funding This paper has been supported by the Deutsche Forschungsgemeinschaft (grant no. 424608044). Open Access funding enabled and organized by Projekt DEAL.

Open Access Dieser Artikel wird unter der Creative Commons Namensnennung 4.0 International Lizenz veröffentlicht, welche die Nutzung, Vervielfältigung, Bearbeitung, Verbreitung und Wiedergabe in jeglichem Medium und Format erlaubt, sofern Sie den/die ursprünglichen Autor(en) und die Quelle ordnungsgemäß nennen, einen Link zur Creative Commons Lizenz beifügen und angeben, ob Änderungen vorgenommen wurden.

Die in diesem Artikel enthaltenen Bilder und sonstiges Drittmaterial unterliegen ebenfalls der genannten Creative Commons Lizenz, sofern sich aus der Abbildungslegende nichts anderes ergibt. Sofern das betreffende Material nicht unter der genannten Creative Commons Lizenz steht und die betreffende Handlung nicht nach gesetzlichen Vorschriften erlaubt ist, ist für die oben aufgeführten Weiterverwendungen des Materials die Einwilligung des jeweiligen Rechteinhabers einzuholen.

Weitere Details zur Lizenz entnehmen Sie bitte der Lizenzinformation auf http://creativecommons.org/licenses/by/4.0/deed.de.

\section{Literatur}

Belina B (2008) Kritische Geographie: Bildet Banden! Einleitung zum Themenheft. Int J Crit Geogr 7(3):335-349

Bloom BS (1972) Taxonomie von Lernzielen im kognitiven Bereich. Beltz, Weinheim, Basel

Brand U, Wissen M (2017) Imperiale Lebensweise: Zur Ausbeutung von Mensch und Natur im globalen Kapitalismus. Oekom, München

Bürk T (2016) Es kann nicht nur darum gehen, dass wir David Harvey unterrichten. Sublurban Z Krit Stadtforsch 4(2/3):233-240

Coy M (2007) Nachhaltigkeit: Ein „,zukunftsfähiges“ Thema für die Geographie? In: GW-Unterricht 107(2007): 1-11. https://www. eduacademy.at/gwb/pluginfile.php/32327/mod_resource/content/ 1/Coy_Nachhaltigkeit_GWU107.pdf. Zugegriffen: 7. Sept. 2020

Getzin S, Singer-Brodowski M (2016) Transformatives Lernen in einer Degrowth-Gesellschaft. J Sci Interfaces 2016(1):33-46

Grober (2015) The discovery of sustainability: the genealogy of a term. In: Enders JC, Remig M (Hrsg) Theories of sustainable development. Routledge, London, New York, S 6-15

Hauenschild K, Bolscho D (2006) Transdisziplinarität als Perspektive für Bildung für nachhaltige Entwicklung in der wissenschaftlichen Ausbildung. In: Hellwig M, Hemker R (Hrsg) Jahrbuch für Nachhaltigkeit 2007. ecotransfer, Münster, S 53-64

Heilgemeir A, Varol A, Termeer B, Schönberg E, Pelger D, Welch Guerra P (2016) „Haltung provozieren“. Ein Gespräch über Möglichkeiten der Kooperation zwischen universitären Lehrveranstaltungen und stadtpolitischen Initiativen. Sublurban Z Krit Stadtforsch 4(2/3):241-248
Hünnemeyer V (2019) Von Generalisten und Spezialisten. Geographen im Beruf. Studie des Deutschen Verbands für Angewandte Geographie e. V. Bargteheide. https://geographie-dvag.de/wpcontent/uploads/2019/09/2019_VonGeneralistenundSpezialisten_ DVAG.pdf. Zugegriffen: 7. Sept. 2020

Kratwohl DR, Bloom BS, Masia BB (1975) Taxonomie von Lernzielen im affektiven Bereich. Beltz, Weinheim, Basel

Krol G-J (2006) Bildung für eine nachhaltige Entwicklung - Ein Beitrag der ökonomischen Perspektive. In: Hiller B, Lange M (Hrsg.) Bildung für eine nachhaltige Entwicklung. Perspektiven für die Umweltbildung. Vorträge und Studien, Bd. 16. Zentrum für Umweltforschung, Münster, S 67-89

Lamker C, Schulze Dieckhoff V, Grotefels S, Mössner S, Schulz C, Wiese-von Ofen I (2018) Mit oder gegen den Strom? Postwachstumsplanung in der Fishbowl. In: pndlonline 2018(2): 1-10. https://orbilu.uni.lu/bitstream/10993/39116/1/lamker-et-al \%202018.pdf. Zugegriffen: 7. Sept. 2020

Lange B, Hülz M, Schmid B, Schulz C (Hrsg) (2020) Postwachstumsgeographien: Raumbezüge diverser und alternativer Ökonomien. transcript, Bielefeld

Leal Filho W (2018) Identifizierung und Überwindung von Barrieren für die Umsetzung einer nachhaltigen Entwicklung an Universitäten: von Studienplänen bis zur Forschung. In: Ebd (Hrsg) Nachhaltigkeit in der Lehre. Eine Herausforderung für Hochschulen. Springer Spektrum, Berlin, Heidelberg, S 1-22

Maschkowsky G, Wanner M (2014) Die Transition-Town-Bewegung - Empowerment für die große Transformation? In: pndlonline 2014(2): 1-11. https://epub.wupperinst.org/frontdoor/deliver/ index/docId/5626/file/5626_Maschkowski.pdf. Zugegriffen: 7. Sept. 2020

Pufé I (2018) Nachhaltigkeit. Bundeszentrale für politische Bildung, Bonn

Schmid B, Smith TSJ (2020) Social transformation and postcapitalist possibility: emerging dialogues between practice theory and diverse economies. Prog Hum Geogr. https://doi.org/10.1177/ 0309132520905642

Schneider P, Gerke G, Folkens L, Busch M (2018) Vernetzung und Weiterentwicklung des Wissenspools zu Nachhaltigkeit in Theorie und Praxis: Umsetzung des Teaching-Research-Practice Nexus an der Hochschule Magdeburg-Stendal. In: Leal Filho W (Hrsg) Nachhaltigkeit in der Lehre. Springer Nature, Wiesbaden, S $107-126$

Schneidewind U (2015) Transformative Wissenschaft: Motor für gute Wissenschaft und lebendige Demokratie. GAIA 24(2):88-91

Schulz C (2012) Post-Wachstums-Ökonomien: (k)ein Thema für die Wirtschaftsgeographie? Z Wirtschgeogr 56(4):264-273

Schwanen T (2018) Thinking complex connections: transition, nexus and geography. Trans Inst Br Geogr 43(2):262-283

Van der Schee J (2003) Kritisches Denken: geographische Denkfähigkeit und bedeutungsvolles Lernen. In: Rolfes M, Uhlenwinkel A (Hrsg) Metzler Handbuch 2.0. Geographieunterricht. Ein Leitfaden für Praxis und Ausbildung. Westermann, Braunschweig, S $105-113$

Vester F (2002) Die Kunst vernetzt zu denken. Ideen und Werkzeuge für einen neuen Umgang mit Komplexität. Ein Bericht an den Club of Rome. dtv (Wissen), München

Zimmermann FM, Zimmermann-Janschitz S (2016) Das Paradoxon der Nachhaltigkeit und warum Nachhaltigkeit in der Geographie (k)eine Rolle spielt. In: Die Welt verstehen - eine geographische Herausforderung. Festschrift der Geographie Innsbruck für Axel Borsdorf, Bd. 40, S 139-162 